\title{
Correction to: Piphillin predicts metagenomic composition and dynamics from DADA2- corrected 16S rDNA sequences
}

Nicole R. Narayan ${ }^{1}$, Thomas Weinmaier', Emilio J. Laserna-Mendieta²,3, Marcus J. Claesson ${ }^{2,3}$, Fergus Shanahan ${ }^{2,4}$, Karim Dabbagh', Shoko Iwai ${ }^{1}$ and Todd Z. DeSantis ${ }^{1 *}$

\section{Correction to: BMC Genomics (2020) 21:56} https://doi.org/10.1186/s12864-019-6427-1

Following the publication of this article [1], the authors reported errors in Figs. 1, 2 and 5. Due to a typesetting error the asterisks denoting significance were missing from the published figures.

The correct figures are reproduced in this Correction article, and the original article has been corrected.

\section{Author details}

'Informatics Department, Second Genome Inc, South San Francisco, California, USA. ${ }^{2}$ APC Microbiome Ireland, University College Cork, Co., Cork, Ireland. ${ }^{3}$ School of Microbiology, University College Cork, Co., Cork, Ireland. ${ }^{4}$ Department of Medicine, University College Cork, Co., Cork, Ireland.

\section{Published online: 31 January 2020}

\section{Reference}

1. Narayan, et al. Piphillin predicts metagenomic composition and dynamics from DADA2- corrected 16S rDNA sequences. BMC Genomics. 2020;21:56. https://doi.org/10.1186/s12864-019-6427-1.

Full list of author information is available at the end of the article 


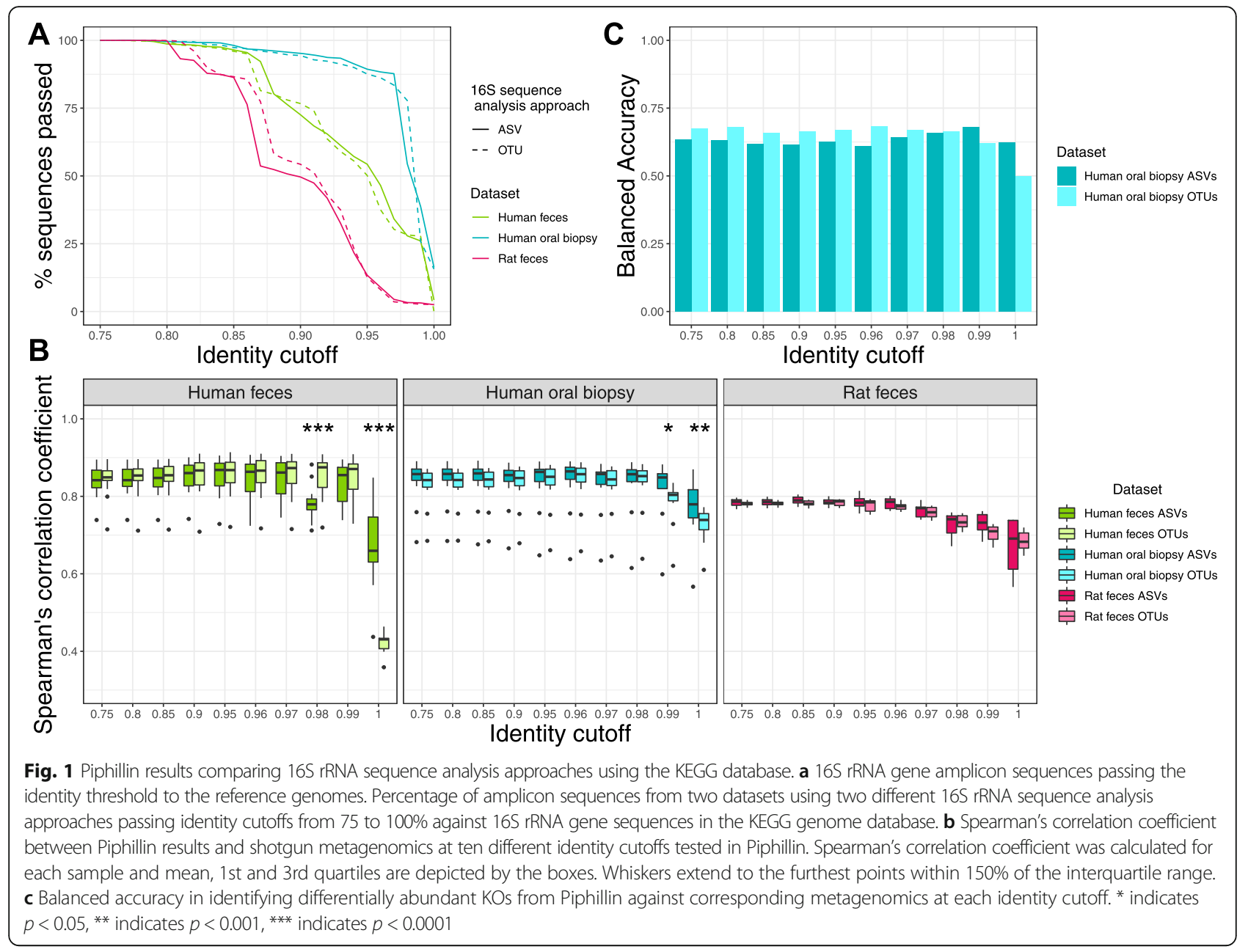



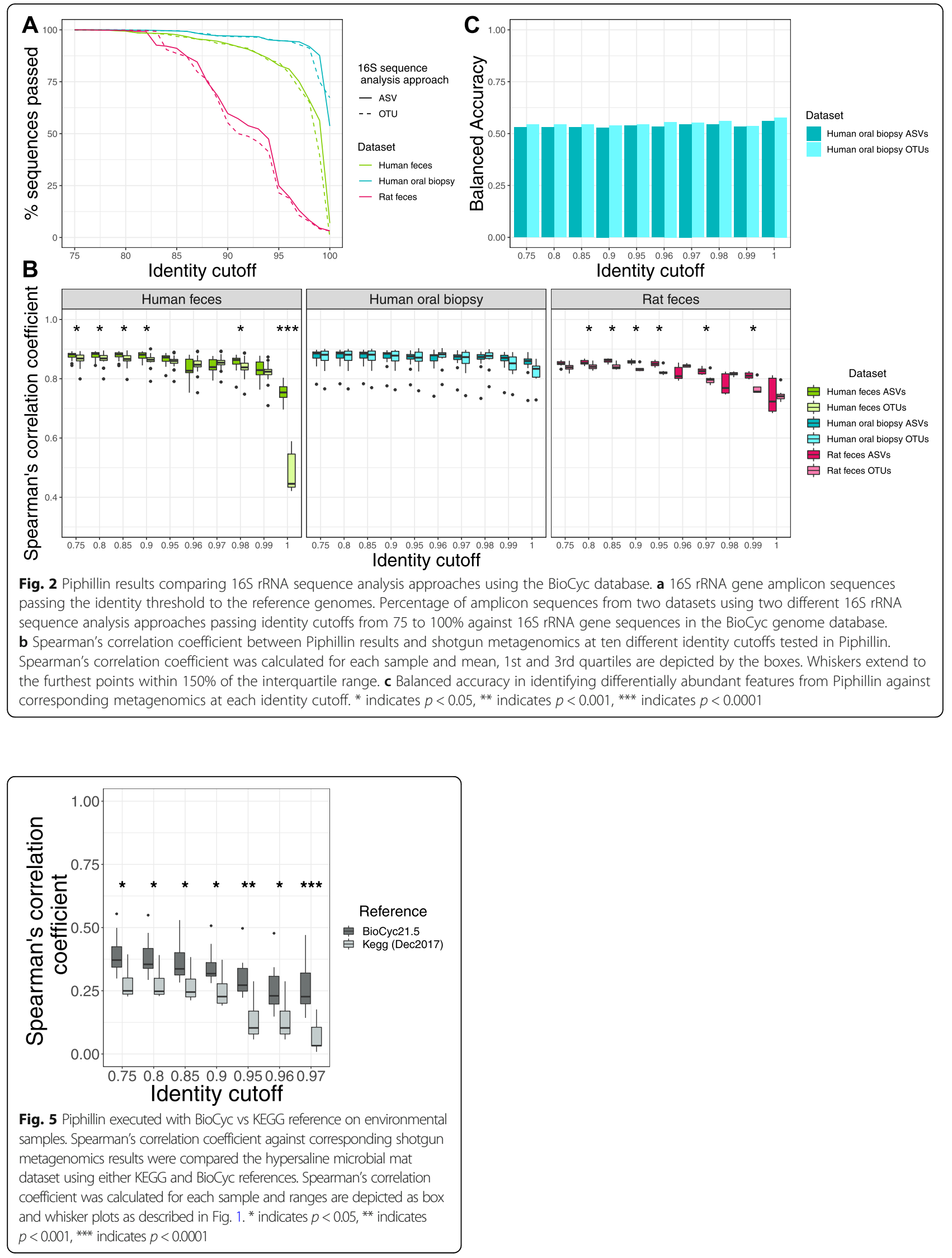